\title{
MEASUREMENTS OF LIGHT FIELDS EMERGING FROM FINE AMPLITUDE GRATINGS
}

\author{
Myun-Sik Kim, ${ }^{*}$ Toralf Scharf, and Hans Peter Herzig \\ Ecole Polytechnique Fédérale de Lausanne (EPFL), Switzerland \\ *myunsik.kim@epfl.ch
}

\begin{abstract}
High resolution amplitude and phase of light fields emerging from a 2-um-period amplitude grating are measured for different wavelengths. The amplitude gratings lead to highly periodic patterns caused by the Talbot effect. Such patterns reach periodicities of a fraction of the grating period. We discuss the effect of wavelengths and the number of diffraction orders participating in the imaging.
\end{abstract}

\section{INTRODUCTION}

Amplitude and phase of light fields emerging from periodic structures, although considered by many researchers, are still an interesting subject of research. Talbot observed the self-imaging effect of a periodic structure in 1836 [1]. Later, Lord Rayleigh showed that its origin is the interference of the diffracted beams and found that the regular repeat distance of self-images, the so-called Talbot Length, can be expressed as

$$
\mathrm{z}_{\mathrm{T}}=2 \Lambda^{2} / \lambda,
$$

where $\Lambda$ is the grating period and $\lambda$ is the wavelength of the incident light [2]. Since smaller gratings have larger diffraction angles given by the diffraction formula as $\sin \theta=\lambda / \Lambda$, high numerical aperture (NA) and high resolution techniques are required to observe these effects for small gratings. This is especially important for grating with periods comparable to the operation wavelength. Moreover, phase measurements need often special techniques to be performed.

In this work, we demonstrate high resolution measurements of amplitude and phase of light fields diffracted by an amplitude grating with $2 \mu \mathrm{m}$ period and using the high resolution interference microscope (HRIM) at several wavelengths. The Talbot effect is observed in the intensity and phase maps and results are discussed. Conditions were light field patterns smaller than the period of the diffraction grating can be observed are evaluated. The newly introduced phase measurements allow accurate evaluation of the Talbot length and provides deeper inside.

\section{EXPERIMENTS}

The HRIM is basically a microscope with objectives that have highest numerical aperture such as $100 \mathrm{X} /$ NA 0.9 combined with a Mach-Zehnder interferometer. Detailed information of system setup is given in references [3, 4]. In the object arm of the interferometer, the $2-\mu \mathrm{m}$-period grating is illuminated at normal incidence with a plane wave. In the reference arm a piezo-electrically driven mirror is mounted to change optical path lengths and the phase distribution of the wave-field is obtained by employing a classical five-frame algorithm [4]. The grating is mounted on a z-axis piezo stage with a scan range of
$500 \mu \mathrm{m}$ and an accuracy of $1 \mathrm{~nm}$. The objects are scanned through the observation plane and three-dimensional (3D) intensity and phase maps are recorded during the measurement. While the amplitude measurement is diffraction limited and has finite spatial resolution, the phase measurement is, in principle, not subjected to resolution issues.

\section{RESULTS AND DISCUSSIONS}

Three different wavelengths, red at $642 \mathrm{~nm}$, green at $532 \mathrm{~nm}$ and blue at $405 \mathrm{~nm}$ are considered and the diffraction angle and the Talbot length are studied. The corresponding $\mathrm{X}-\mathrm{Z}$ slices of the measured 3D intensity maps for three wavelengths are shown in Fig. 1. The propagation direction is $z$. One observes self-images at $\mathrm{z}_{\mathrm{T}}$ and shifted pattern by half period at the half of the Talbot length $\left(\mathrm{z}_{\mathrm{T}} / 2\right)$ as expected from theory. The Talbot lengths are calculated by using Eq. (1) to be $12.5 \mu \mathrm{m}$ for $642 \mathrm{~nm}, 15 \mu \mathrm{m}$ for $532 \mathrm{~nm}$, and $19.8 \mu \mathrm{m}$ for $405 \mathrm{~nm}$. The visibility of the smaller fractions of the Talbot images differs depending on the illuminating wavelength because the number of collected diffraction orders by the measurement objective varies for different wavelengths.

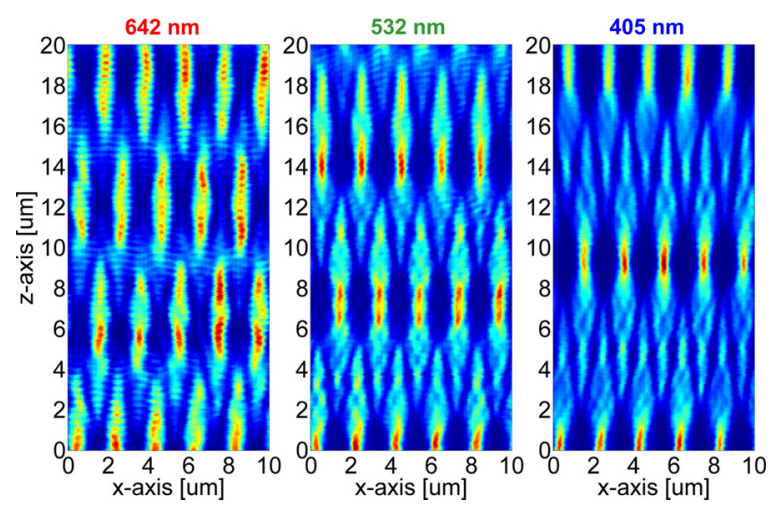

Fig. 1. The $x-z$ slices of the measured $3 D$ intensity map of $10 \times 20 \mu \mathrm{m}^{2}$ : (a) $642 \mathrm{~nm}$, (b) $532 \mathrm{~nm}$, and (c) $405 \mathrm{~nm}$.

For a 2- $\mu \mathrm{m}$-period grating at normal incidence one finds the angle for the first diffraction order as $18.7^{\circ}$ for $642 \mathrm{~nm}$, $15.4^{\circ}$ for $532 \mathrm{~nm}$, and $11.7^{\circ}$ for $405 \mathrm{~nm}$. The observing objective has a numerical aperture of $\mathrm{NA}=0.9$ corresponding to a maximum acceptance angle of $64^{\circ}$. Hence, diffraction from orders 0 to \pm 3 can be collected by this objective at $642 \mathrm{~nm}$ illumination. For $532 \mathrm{~nm}$ and $405 \mathrm{~nm}$, higher diffraction orders from 0 to \pm 4 and 0 to \pm 5 contribute, respectively. This can be verified by observing the back focal plane of the objective with the help of a telescope or Bertrand lens. Figure 2 shows images of the back focal plane of the objective and the bright spots are 
the diffraction orders. In practice, intensity falls off for large angles and less diffraction orders might be collected and certain experimental errors limit the NA of the objective to be smaller than 0.9. However, the number of diffraction orders gets fewer as the wavelength increases, as shown in Fig. 2. Better contrast and higher resolution are obtained for the intensity measurement at $405 \mathrm{~nm}$ thanks to the contributed higher orders.
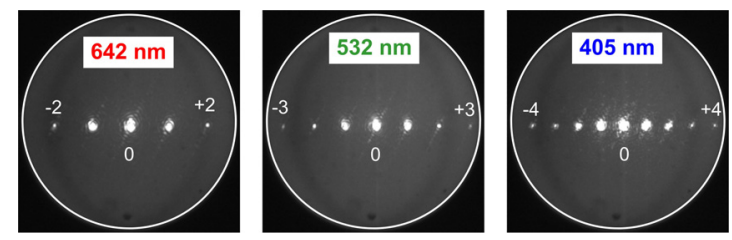

Fig. 2. Images of the back focal plane of the $100 \mathrm{X} / \mathrm{NA} 0.9$ objective lens. The grating is illuminated by a plane wave of (a) $642 \mathrm{~nm}$, (b) $532 \mathrm{~nm}$, (c) $405 \mathrm{~nm}$. White circle shows the exit pupil and the 0 order as well as the highest diffraction order which are indicated.

To obtain more information the phase of the emerging fields are investigated by applying the aforementioned interferometric technique. Figures 3(a)-3(c) show the $x-z$ slices of the measured 3D phase maps for the three wavelengths. At the multiple of the half of the Talbot length $\left(\mathrm{z}_{\mathrm{T}} / 2\right)$, planes with particular phase structures, such as phase singularities, are found. Because of the extremely low intensity near the plane of the singularities, measurement noise is amplified. Compared to the intensity measurements, the phase maps are more detailed. More precise evaluations of the Talbot length can be done by measuring the distance between the planes where phase singularities occur.

\section{CONCLUSIONS}

We present high resolution amplitude and phase measurements of light fields emerging from the $2-\mu \mathrm{m}$ period amplitude grating measured with a high resolution interference microscope. The number of the collected diffraction orders determines resolution and visibility of the smaller fractions of the Talbot images in the intensity and phase measurements. The phase maps allow more accurate measurements of the Talbot length by using the position of singularities because the phase field is not subjected to a resolution limit.

\section{ACKNOWLEDGMENT}

The research leading to these results has received funding from the European Community's Seventh Framework Programme FP7-ICT-2007-2 under grant agreement No. 224226.

\section{REFERENCES}

[1] F. Talbot, "Facts relating to optical science. IV" Philos. Mag. 9, 401-407, 1836

[2] L. Rayleigh, "On copying diffraction gratings and some phenomena connected therewith" Philos. Mag. 11, 196-205, 1881

[3] C. Rockstuhl, et al., "High Resolution Interference Microscopy: A Tool for Probing Optical Waves in the FarField on a Nanometric Length Scale" Current Nanoscience 2, 337-350,2006

[4] M.-S. Kim, et al., "Amplitude and Phase Measurements of Highly Focused Light in Optical Data Storage Systems" accepted for publication in Jpn. J. Appl. Phys., 2010

[5] J. Schwider, et al., "Digital wave-front measuring interferometry: some systematic error sources" Appl. Opt. $22,3421-3432,1983$
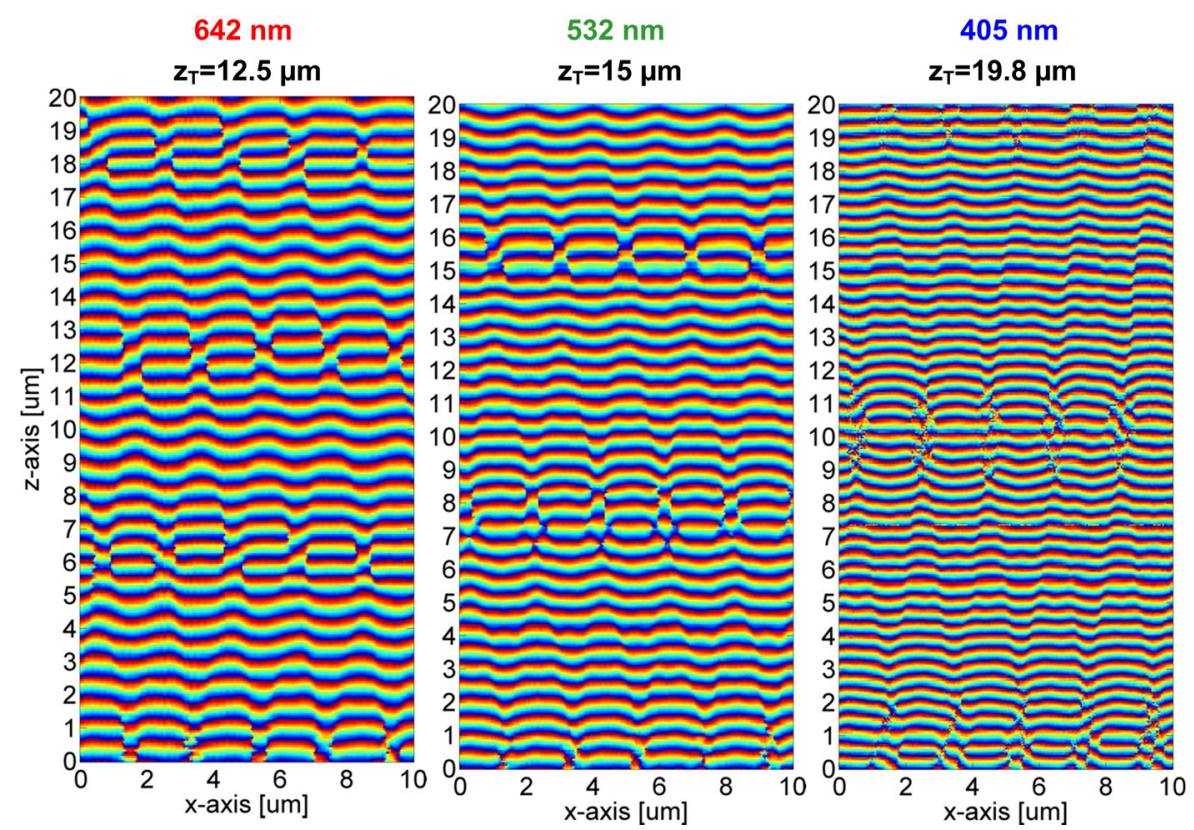

Fig. 3. The $x-z$ slices of the measured 3D phase map of $10 x 20 \mu m^{2}$ : (a) $642 \mathrm{~nm}$, (b) $532 \mathrm{~nm}$, and (c) $405 \mathrm{~nm}$. At the multiple of the half Talbot length $\left(\mathrm{z}_{\mathrm{T}} / 2\right)$, the self-images of the plane wave surrounded by the phase singularities are observed. 